International Braz J Urol

Vol. 35 (1): 84-89, January - February, 2009

\title{
Urethral Catheter Insertion Forces: A Comparison of Experience and Training
}

\author{
Benjamin K. Canales, Derek Weiland, Scott Reardon, Manoj Monga
}

Department of Urology (BKC), University of Florida, Gainsville, Florida, USA and Department of Urology (DW, SR, MM), University of Minnesota, Minneapolis, Minnesota, USA

\begin{abstract}
Purpose: This study was undertaken to evaluate the insertion forces utilized during simulated placement of a urethral catheter by healthcare individuals with a variety of catheter experience.

Materials and Methods: A 21F urethral catheter was mounted to a metal spring. Participants were asked to press the tubing spring against a force gauge and stop when they met a level of resistance that would typically make them terminate a catheter placement. Simulated catheter insertion was repeated fives times, and peak compression forces were recorded. Healthcare professionals were divided into six groups according to their title: urology staff, non-urology staff, urology resident/ fellow, non-urology resident/ fellow, medical student, and registered nurse.

Results: A total of fifty-seven healthcare professionals participated in the study. Urology staff $(n=6)$ had the lowest average insertion force for any group at $6.8 \pm 2.0$ Newtons $(\mathrm{N})$. Medical students $(\mathrm{n}=10)$ had the least amount of experience ( $1 \pm 0$ years) and the highest average insertion force range of $10.1 \pm 3.7 \mathrm{~N}$. Health care workers with greater than 25 years experience used significantly less force during catheter insertions $(4.9 \pm 1.8 \mathrm{~N})$ compared to all groups $(\mathrm{p}<0.01)$.

Conclusions: We propose the maximum force that should be utilized during urethral catheter insertion is 5 Newtons. This force deserves validation in a larger population and should be considered when designing urethral catheters or creating catheter simulators. Understanding urethral catheter insertion forces may also aid in establishing competency parameters for health care professionals in training.
\end{abstract}

Key words: urinary catheterization; urethral catheter; educational models

Int Braz J Urol. 2009; 35: 84-9

\section{INTRODUCTION}

The insertion of a urethral catheter is one of the most commonly performed hospital procedures. For the year 2000, the Center for Disease Control estimated that over five million urethral catheterizations were attempted in the United States (1). In most hospitals, this procedure is performed without a standardized training protocol and by a variety of healthcare workers with varying degrees of experience. We hypothesized that we could establish competency parameters for professionals in-training by measuring urethral catheter exertion forces and comparing healthcare provider experience. In addition, we would gather information that may affect not only the future design of catheters and virtual simulators but also the rate of urethral trauma and stricture.

\section{MATERIALS AND METHODS}

Healthcare personnel at the Veterans Affairs Medical Center and the University of Minnesota in 
Minneapolis, MN, USA, were invited to participate in our study if they could be stratified into one of six groups: Group 1 - Urologist MD; Group 2 - NonUrologist MD; Group 3 - Urology Resident / Fellow MD; Group 4 - Non-Urology Resident / Fellow MD; Group 5 - Medical Student; and Group 6 - Registered Nurse. Participants were asked, "Push in the catheter until you feel a level of resistance that would make you stop if you were putting in a real urethral catheter". Participants held the catheter at the same marked area and were instructed and monitored by the same two researchers to ensure procedure conformity. The procedure was repeated five times serially by all groups.

The tube used in simulation was a polyolefin catheter $(21 \mathrm{~F}$ outer circumference, $9 \mathrm{~F}$ inner circumference) with a metal spring (7/32" x 1 " x 0.28 " wire thickness) mounted on the distal end of a compression force gauge (Extech ${ }^{\mathrm{TM}}$ Digital Force Gauge - model 475040) (Figure-1). Peak compression forces in Newtons $(\mathrm{N})$ were recorded, and participants were blinded to their own results.

The statistical software package SAS was used for all calculations (SAS Institute Inc., Cary, $\mathrm{NC}$, Version 9.0). Group mean comparisons were

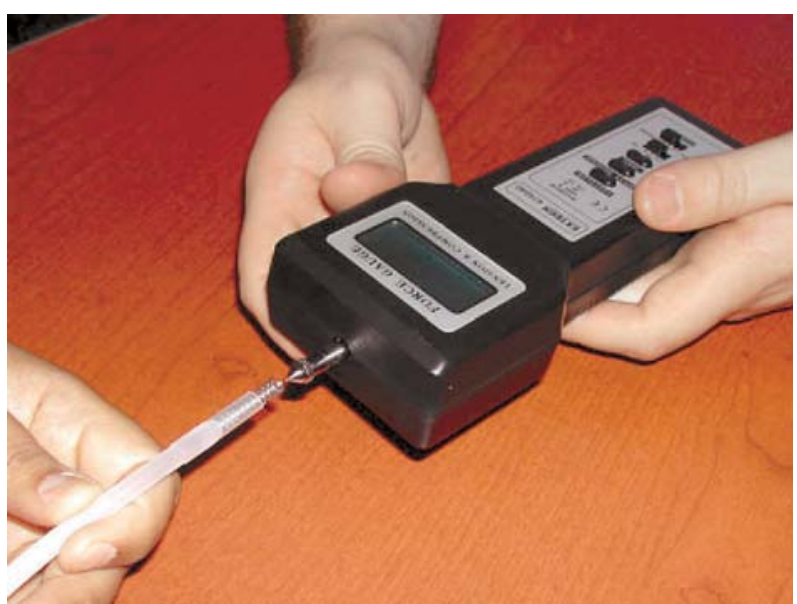

Figure 1 - Photograph of the hand-position taken by a lefthanded individual being tested on an Extech ${ }^{T M}$ Digital Force Gauge - model 475040. Participants were asked to hold the silicone portion and press on the tubing similar to advancing a catheter. A plastic shield (not shown) was placed between the force gauge and catheter to blind participants to their results. calculated by an unpaired, two-sided, Student ttest. Analysis of variance (ANOVA) was applied to compare different groups with respect to continuous variables. Change in force over years of experience was estimated by regressing the force applied by each subject and obtaining a best fit line by linear regression. Results were considered significant if the $\mathrm{p}$-value was $<0.05$.

\section{RESULTS}

Fifty-seven healthcare workers participated in this study (Figure-2). Individual urethral catheter insertion force was averaged by group and ranged from 6.8 to $10.1 \mathrm{~N}$. Urologists (Group-1) had the lowest average force insertion forces $(6.8 \mathrm{~N} \pm 2.0$ $\mathrm{N}$ ), while medical students (Group-5) had the highest average insertion forces $(10.1 \mathrm{~N} \pm 3.7 \mathrm{~N})$. The difference in the amount of simulated force used to insert a catheter was significantly higher for the medical students compared to every other group $(\mathrm{p}<0.01)$. The difference in $p$ values was also significant when comparing urologists to urology residents (Group-3, $\mathrm{p}=0.03$ ) and registered nurses (Group-6, $\mathrm{p}<0.01$ ).

To evaluate for subject-expectancy effect, one way ANOVA was performed comparing force versus attempt for attempts \#1 - 5 (Figure-3). No statistically significant difference was found between means of groups (range $8.14 \mathrm{~N}-8.62 \mathrm{~N} ; \mathrm{p}=0.96$ ). Experience was then plotted independent of medical group in a bivariate fit graph versus force, and results were examined using ANOVA (Figure-4). In this linear correlation, participant experience alone $(\mathrm{r}=$ - 0.78) explained approximately $78 \%$ of the observed variation in force $(\mathrm{p}<0.001)$. Health care workers with more than 25 years experience $(n=9)$ had the lowest average force insertion forces $(4.9 \mathrm{~N} \pm 1.8 \mathrm{~N})$ compared to those with less than 25 years experience $(8.4 \pm 2.5 \mathrm{~N}, \mathrm{p}=<0.01)$.

\section{COMMENTS}

The urethral catheter is an instrument as old as the field of urology. Evidence of catheter use in Greece can be found in the Hippocratic Writings ( 400 BC) 

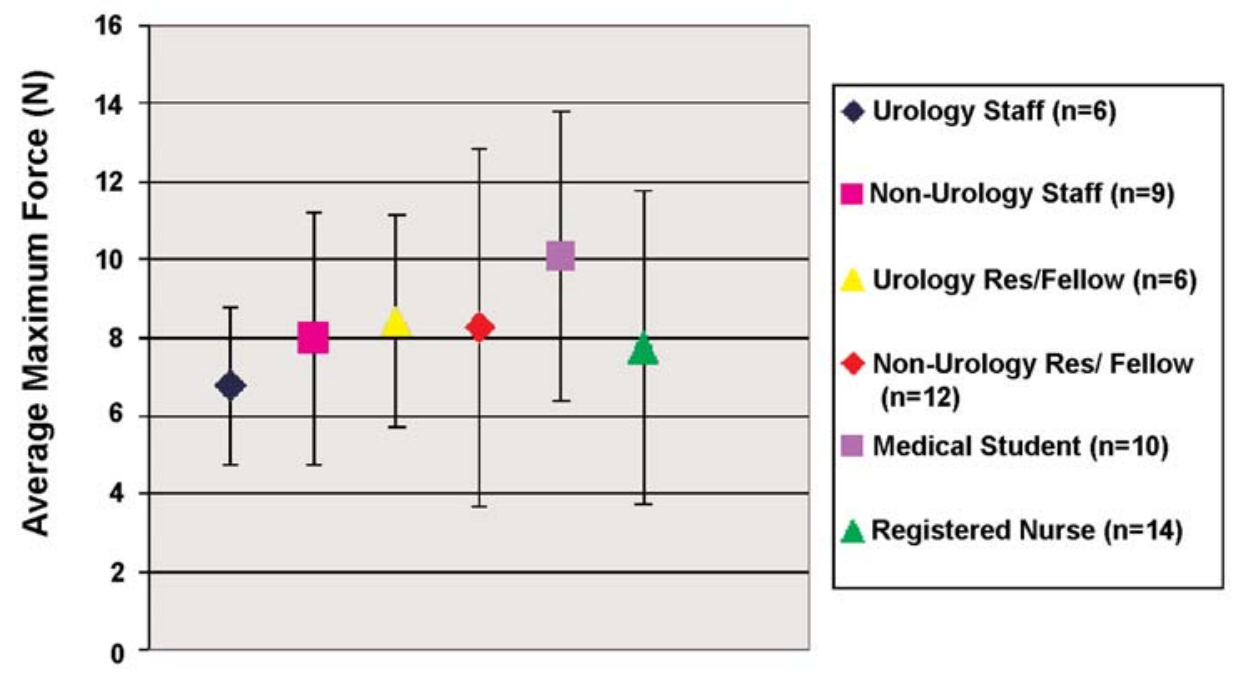

Figure 2 - Average urethral catheter insertion force in Newtons, by group.

(2). In 1929, Dr. Frederick E.B. Foley described the first modern urethral catheter by dipping coagulating latex onto metal forms to create a dual-port balloon catheter (3). Components of modern urethral catheters have evolved into a combination of silicone and latex-free rubber (mixed in varying proportions to vary catheter rigidity) coated with an elastomer to aid insertion (4).

Despite the publications of a nurse clinical practice guideline (5) and the impact of nursing educa-

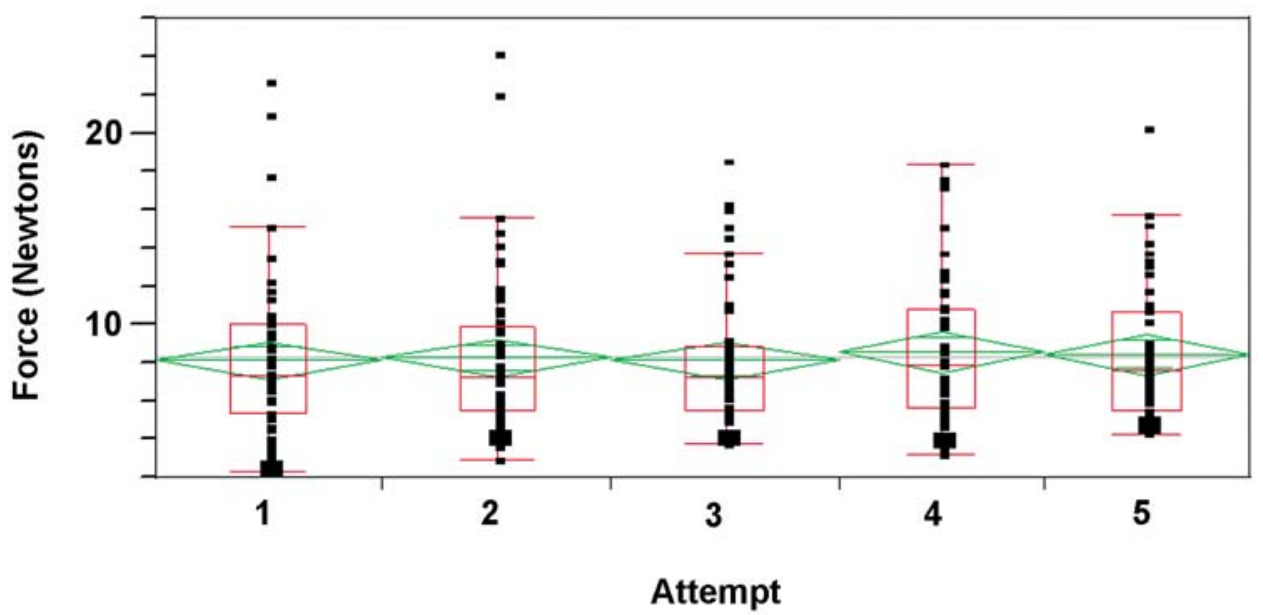

Figure 3 - One way analysis of variance comparing force (y-axis) versus attempt ( $x$-axis). Mean force (green diamond lines) by attempt ranged from $8.14 \mathrm{~N}-8.62 \mathrm{~N}(p=0.96)$. 


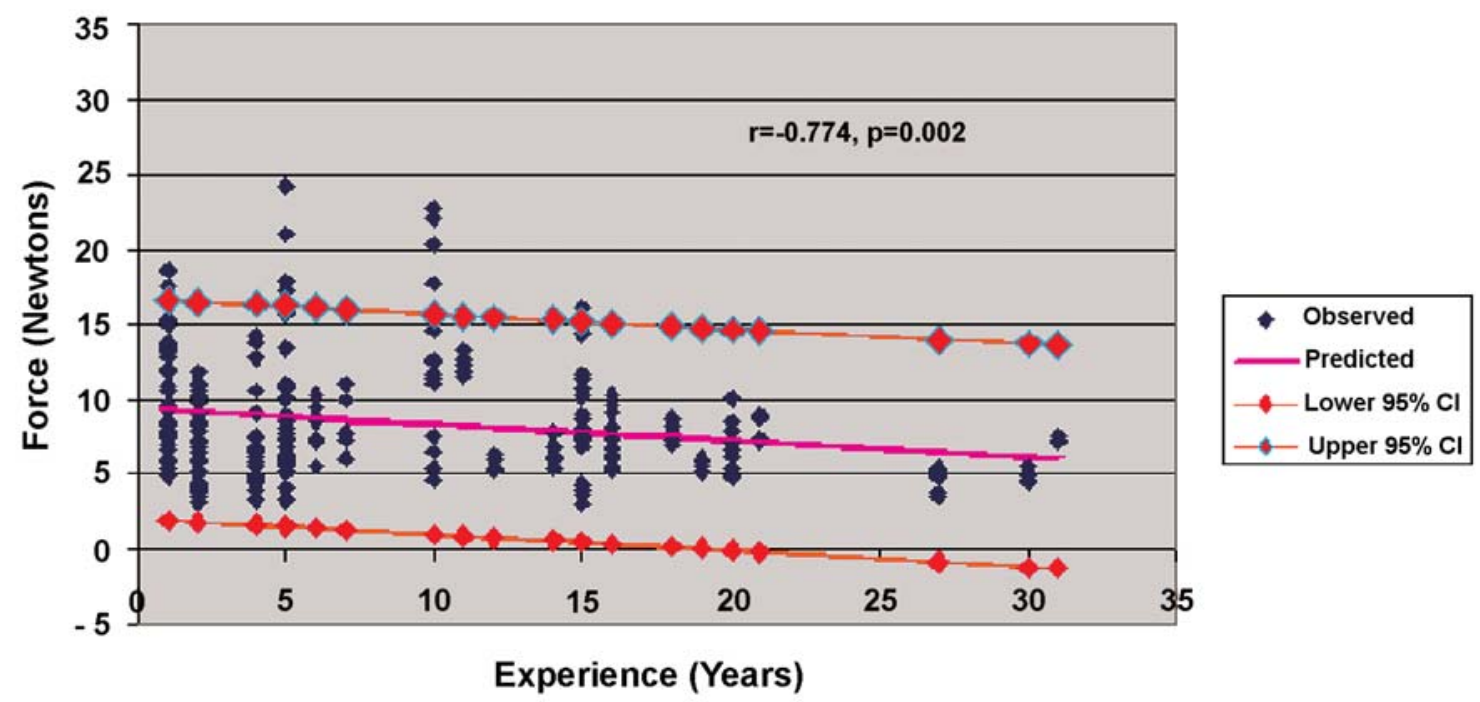

Figure 4 - Simple linear regression plot of forces (observed and predicted) and participant experience with 95\% confidence intervals for the mean. $r$ = correlation coefficient.

tional programs (6), no standardized method for training health care workers in catheter placement exists. Most new health care employees, whether they are students or residents, are guided through the process of patient preparation and catheterization by someone with catheter experience. During the actual procedure, however, only the health care worker advancing the catheter can feel the resistance given by the catheter. Although improvements in catheter design and composition have occurred, iatrogenic urethral injury, in particular urethral stricture, continues to occur far too commonly $(7,8)$. Fenton et al. found that urethral catheter placement was the cause of approximately $30 \%$ of all urethral stricture disease (9). In addition, urethral-rectal fistulas, urethral perforation, prostatic bleeding requiring surgical intervention, and bladder perforation have all been reported as consequences of improperly inserted urethral catheters (10-12). Therefore, we attempted to generate a "normal curve" of catheter forces that could be used for modeling purposes and potential competency parameters.

From a design standpoint, it is important to emphasize that we have measured only one element of catheter insertion: force used. Because our method of simulation does not involve resistance, we chose to use the polymer "polyolefin" as it is more firm than a silicone or latex catheter and does not buckle prior to the participant reaching the point of maximal force. Analysis by attempt (Figure-3) indicated that no more force was applied on the first attempt than on the last attempt, suggesting that both the material used and the study design yielded reproducible and precise results.

Intuitively, it makes sense that a properly placed catheter should not require a great deal of force to traverse the urethra. In support of this, the experienced urologists had the lowest catheter insertion forces of all our groups $(6.8 \pm 2.0 \mathrm{~N})$. More surprising was the finding that health care workers with more than 25 years experience had even lower forces $(4.9 \pm 1.8 \mathrm{~N})$ with statistical significance. Because of this, we propose that the range of $4.9 \pm 1.8 \mathrm{~N}$ be considered the standard-of-care model in regards to urethral catheter insertion force. Future clinical studies should evaluate not only force but also the ability for providers to appreciate and adapt to variances in a clinical presentation, such as catheter resistance, anatomy, bloody return, or patient discomfort.

Several catheter methods of simulation have been devised, but no simulation method fully recreates the sense of catheterization of the female or male. Despite their shortcomings, catheter simulators have been shown to reduce both risk and pain experienced by patients in addition to avoiding urethral injuries 
(13). Since many U.S. medical schools have the insertion of a urethral catheter as a core clinical competency (14), simulators could likely help students learn this core concept before actually practicing on a real patient. As the use of simulated medical trainers rises in this country, it is our hope that this data can be used to help industry sector and medical programs in designing simulation devices that give feedback during urethral catheter insertion. From a cost standpoint, Morgan et al. showed that medical simulator training devices are worth the extra expense in regards to both student and faculty satisfaction (15). Although most of the medical simulator published data involves medical students, this technology could easily be applied to nursing students (who are much more likely to place routine catheters than physicians are), new surgical or medical residents, and/or as competency testing for physicians.

Our study has some limitations. Though utilization of an in vitro model allows standardization of technique and measurement, it does not account for signs of excess force that could be noted in a clinical trial, such as patient reporting of discomfort, blood at the urethral meatus, or catheter tip resistance. In addition to force, urethral injury rates may increase by higher catheterization intervals or in high risk male populations, such as benign prostatic hyperplasia or prostate cancer (6). Polyolefin material is slightly stiffer than silicone and latex materials used in catheters and was selected specifically so the catheter would not buckle during testing. It would be of value to validate the force range defined in this study in a clinical trial of a larger group of providers using commercially available catheters.

\section{CONCLUSION}

In conclusion, as health care workers acquire more experience, significantly less force is used during urethral catheter insertions. Based on our findings, we propose that the maximum force that should be utilized during urethral catheter insertion is 5 Newtons. This force should be considered the "upper limit" utilized for urethral catheter insertion. Future validation in larger populations, such as measurements in cadavers or anesthetized patients undergoing non-urologic surgery, would be warranted to evaluate the range of forces used during normal circumstances. However, clinical measurements of this type may not necessarily help refine the "upper limit" that should be avoided unless they are collected from experienced practitioners. Overall, understanding urethral catheter insertion forces may aid in the design of future catheters, in the creation of catheter simulators, and in establishing competency parameters for health care professionals during training and/or recertification.

\section{ACKNOWLEDGEMENT}

To Dr. John J. Carlow who assisted in the statistical analysis.

\section{CONFLICT OF INTEREST}

Financial support by PercSys ${ }^{\circledR}$ (Percutaneous Systems, Inc.; Mountain View, CA)

\section{REFERENCES}

1. Maki DG, Tambyah PA: Engineering out the risk for infection with urinary catheters. Emerg Infect Dis. 2001; 7: 342-7.

2. Moog FP, Karenberg A, Moll F: The catheter and its use from Hippocrates to Galen. J Urol. 2005; 174: 1196-8.

3. Newman DK: Managing indwelling urethral catheters. Ostomy Wound Manage. 1998; 44: 26-32.

4. Carr HA: A short history of the Foley catheter: from handmade instrument to infection-prevention device. J Endourol. 2000; 14: 5-8.

5. Clinical Practice Guidelines Task Force; Society of Urologic Nurses and Associates. Male urethral catheterization. Urol Nurs. 2006; 26: 315-7.

6. Kashefi C, Messer K, Barden R, Sexton C, Parsons JK: Incidence and prevention of iatrogenic urethral injuries. J Urol. 2008; 179: 2254-7; discussion 2257-8.

7. Dobrowolski ZF, Weglarz W, Jakubik P, Lipczynski W, Dobrowolska B: Treatment of posterior and anterior urethral trauma. BJU Int. 2002; 89: 752-4.

8. Igawa Y, Wyndaele JJ, Nishizawa O: Catheterization: possible complications and their prevention and treatment. Int J Urol. 2008; 15: 481-5. 
9. Fenton AS, Morey AF, Aviles R, Garcia CR: Anterior urethral strictures: etiology and characteristics. Urology. 2005; 65: 1055-8.

10. Nouri M, Tazi K, el Fassi J, Ibn Attya A, Hachimi M, Lakrissa A: Treatment of urethro-rectal fistulas. Apropos of 5 cases. Prog Urol. 1999; 9: 137-41.

11. Donovski L, Ormanov I, Ziad Sh, Farakh N: A case of iatrogenic rupture of the urethra with a retrovesical urinoma. Khirurgiia (Sofiia). 1989; 42: 72-4.

12. Basha M, Subhani M, Mersal A, Saedi SA, Balfe JW: Urinary bladder perforation in a premature infant with Down syndrome. Pediatr Nephrol. 2003; 18: 118990.

\section{Correspondence address:}

Dr. Benjamin K. Canales

Dept of Urology, University of Florida

1600 SW Archer Rd, Rm N-213

PO Box 100247

Gainesville, FL, 32610-0247, USA

Fax: + 1352 273-7515

E-mail: benjamin.canales@urology.ufl.edu
13. Gordon JA, Wilkerson WM, Shaffer DW, Armstrong EG: "Practicing" medicine without risk: students' and educators' responses to high-fidelity patient simulation. Acad Med. 2001; 76: 469-72.

14. Sanders CW, Edwards JC, Burdenski TK: A survey of basic technical skills of medical students. Acad Med. 2004; 79: 873-5.

15. Morgan PJ, Cleave-Hogg DM: Cost and resource implications of undergraduate simulator-based education. Can J Anaesth. 2001; 48: 827-8.

Accepted after revision:

October 13, 2008

\section{EDITORIAL COMMENT}

This work is a highly valuable unprecedented initiative towards systemization of urethral catheter insertion. Although urethral catheter insertion is a common procedure, to date there is no definitive standardization.

The use of diverse groups in the study serves to show that aptitude in catheter placement implicates an extremely long learning curve for all healthcare providers, independent of their specialization. U1- timately, this study shows that even if other factors can lead to late complications, the force used in the placement of the catheter is the principal factor leading to acute complications.

Lastly, this study serves as a starting point for creation of catheter simulators designed for healthcare workers. However, as documented by the authors, more studies must be conducted in order to validate all these data.
Dr. João P. Martins de Carvalho Section of Urology Fluminense Federal University (UFF) Niteroi, Rio de Janeiro, Brazil E-mail: carvalho.jpm@gmail.com 